\title{
CORRELAÇÃO LINEAR E ESPACIAL ENTRE A PRODUTIVIDADE DE FEIJÃO E A POROSIDADE DE UM LATOSSOLO VERMELHO DE SELVÍRIA (MS) ${ }^{(1)}$
}

\author{
Marcio Mahmoud Megda ${ }^{(2)}$, Morel de Passos e Carvalho( ${ }^{(3)}$, Michele \\ Xavier Vieira ${ }^{(2)}$, Marcelo Andreotti ${ }^{(3)}$ \& Elaine Cristina Pereira ${ }^{(2)}$
}

\begin{abstract}
RESUMO
A porosidade do solo exerce grande influência sobre o crescimento e desenvolvimento vegetal, uma vez que o crescimento das raízes, tal como a produtividade das culturas, é limitado pela profundidade que atingem. Portanto, o objetivo deste trabalho foi estudar a variabilidade espacial e as correlações lineares entre a produtividade de feijão e a porosidade do solo. No ano de 2004/ 2005, no município de Selvíria, MS, foram analisadas a produtividade de grãos de feijão ( $P G)$, cultivar IAC Carioca, irrigado, a macroporosidade (MA), a microporosidade (MI) e a porosidade total (PT) do solo em quatro profundidades: $1(0,0-0,10) ; 2(0,10-0,20) ; 3(0,20-0,30)$ e $4(0,30-0,40 \mathrm{~m})$, num Latossolo Vermelho distroférrico. As amostras de solo e planta foram coletadas em uma malha geoestatística com 75 pontos espaçados de $10 \times 10 \mathrm{~m}$, mais 60 pontos de quatro malhas de refinamento numa área de $50 \times 150 \mathrm{~m}$. Os atributos estudados, além de não terem variado aleatoriamente, apresentaram média e baixa variabilidades. Seguiram padrões espaciais bem definidos, com alcances entre 11,70 e $104,40 \mathrm{~m}$. A correlação linear entre o atributo da planta e os do solo, em função do elevado número de observações, foi baixa. As de melhor correlação linear com a PG foram a MA1, MI1 e a PT3. Entretanto, do ponto de vista espacial, houve excelente correlação inversa entre a PG e a \#PT2. Assim, nos sítios onde a \#PT2 diminuiu $\left(0,030-0,045 \mathrm{~m}^{3} \mathrm{~m}^{-3}\right)$, a $\mathrm{PG}$ variou entre 2.173 e $3.529 \mathrm{~kg} \mathrm{ha}^{-1}$. Já naqueles onde aumentou $\left(0,045-0,076 \mathrm{~m}^{3} \mathrm{~m}^{-3}\right)$, a PG ficou entre $1.630-2.173 \mathrm{~kg} \mathrm{ha}^{-1}$. Assim, a porosidade total do solo, quando avaliada na camada de 0,10-0,20 m (\#PT2), indicou a importância do contato raiz/solo e, de modo inverso, apresentou satisfatório
\end{abstract}

\footnotetext{
(1) Recebido para publicação em julho de 2007 e aprovado em novembro de 2007.

(2) Graduando em Engenharia Agronômica, Universidade Estadual Paulista "Júlio de Mesquita Filho" - UNESP. Campus de Ilha Solteira, Avenida Brasil 56, Centro, Caixa Postal 31, CEP 15385-000 Ilha Solteira (SP). E-mails: marcio_agr@yahoo.com.br, micheleagronomia@yahoo.com.br, ecpereira@aluno.feis.unesp.br

${ }^{(3)}$ Professor Livre-Docente - Universidade Estadual Paulista “Júlio de Mesquita Filho”, UNESP. E-mails: morel@agr.feis.unesp.br; mpcarva53@yahoo.com.br; dreotti@agr.feis.unesp.br
} 
indicador da qualidade física do solo estudado, quando destinado à produtividade de grãos de feijão irrigado.

Termos de indexação: variabilidade espacial, manejo do solo, plantio direto, Phaseolus vulgaris L.

\title{
SUMMARY: LINEAR AND SPATIAL CORRELATION BETWEEN COMMON BEAN YIELD AND POROSITY OF A RED LATOSOL IN SELVÍRIA (MS), BRAZIL
}

\begin{abstract}
Soil porosity influences plant development since root growth and crop yield are determined by the root depth. The objective of this study was to investigate the linear and spatial variability and correlations between common bean yield and soil porosity. The bean grain yield of the irrigated cultivar Carioca IAC was analyzed in the growing season 2004/2005, in Selviria-MS, as well as macroporosity (MA), microporosity (MI) and total porosity (TP), in a Dystroferric Red Latosol, at four depths: $1(0.0-0.10 \mathrm{~m}), 2(0.10-0.20 \mathrm{~m})$, $3(0.20-0.30 \mathrm{~m})$ and $4(0.30-0.40 \mathrm{~m})$. Soil and plant data were collected in a geostatistical grid with 135 points spaced $10 \mathrm{~m}$ apart, covering an area of $50 \times 150 \mathrm{~m}$. The data of the studied attributes did not vary randomly and the values were intermediate to low. They followed well-defined spatial standards, reaching between 11.70-104.40 m. On the other hand, the linear correlation between the plant and soil attributes was low, due to the high number of observations. Grain yield had the best linear correlations with MA1b, MI1 and TP3. From the spatial point of view, the inverse correlation between PG and \#TP2 was outstanding. At the sites where \#TP2 diminished $\left(0.030-0.045 \mathrm{~m}^{3} \mathrm{~m}^{-3}\right)$ the yield varied from 2,173 to $3,529 \mathrm{~kg} \mathrm{ha}^{-1}$ and where it increased $\left(0.045-0.076 \mathrm{~m}^{3} \mathrm{~m}^{-3}\right)$, the yield was between 1,630 and $2,173 \mathrm{~kg} \mathrm{ha}^{-1}$. Therefore, the total soil porosity, evaluated in the 0.10-0.20 m layer (\#TP2), indicated the importance of the contact root/soil and was in turn a satisfactory indicator of soil physical quality, with a view to the grain yield of irrigated common bean.
\end{abstract}

Index terms: spatial variability, soil management, no-tillage, Phaseolus vulgaris $L$.

\section{INTRODUÇÃO}

A cultura do feijão constitui-se numa das mais importantes culturas agrícolas do Brasil, não só pela área cultivada e pelo valor da produção como também por se tratar da principal fonte de proteína das camadas sociais de menor poder aquisitivo. Juntamente com o arroz, o milho e a mandioca, o feijão representa a base da alimentação humana. Trata-se de uma importante cultura de inverno, utilizada tanto em sucessão quanto em rotação com outras, pois proporciona boa rentabilidade ao agricultor, além de gerar empregos.

A baixa produtividade de grãos para esta cultura no Brasil (950 kg ha-1), segundo Embrapa (2007), é decorrente, em parte, da falta de cultivares mais produtivos, com acentuada estabilidade e adequada capacidade de adaptação. Nos últimos 15 anos, a produtividade mundial de feijão foi de $600 \mathrm{~kg} \mathrm{ha}^{-1}$ segundo FAO (2007), considerado baixo por ter a cultura um potencial para rendimento superior a $3.500 \mathrm{~kg} \mathrm{ha}^{-1}$ (FNP, 2007). Contudo, em regiões que a agricultura é mais tecnificada, como Estado de São Paulo, o rendimento médio já ultrapassa $1.200 \mathrm{~kg} \mathrm{ha}^{-1}$ (Camargo et al., 2001).

Muitos estudos relativos à compactação do solo convergem para o fato de que, com seu aumento, desencadearia, no geral, uma diminuição da produtividade agrícola. Assim, de acordo com Goedert (2002), a diminuição dos macroporos constitui um tema de crescente importância em face do aumento da mecanização nas atividades agrícolas, que acarreta alteração no arranjo das partículas do solo, tornandoo mais denso. Os principais efeitos negativos deste fato são o aumento da resistência mecânica ao crescimento radicular, a redução da aeração (macroporos) e a disponibilidade de água (microporos) e de nutrientes, e, conseqüentemente, decréscimo na produtividade agrícola. Trabalhos têm mostrado que, quando a macroporosidade do solo é reduzida a valor inferior a $0,15 \mathrm{~m}^{3} \mathrm{~m}^{-3}$, o crescimento das raízes é prejudicado. Neste caso, somente a partir de densidade do solo equivalente a $1,4 \mathrm{~kg} \mathrm{dm}^{-3}$ ocorreria problema para as raízes. Além disso, ao diminuir a macroporosidade, segundo Kertzmann (1996), parte 
significativa da água fica retida nos microporos sob altas tensões e, portanto, indisponível às plantas.

Atualmente, os estudos geoestatísticos vêm contribuindo muito com a pesquisa da variabilidade espacial, principalmente aquela voltada aos atributos físicos do solo e da produtividade vegetal (Carvalho et al., 2002; Santos et al., 2006). Assim, com o objetivo de estudar a variabilidade espacial e as correlações lineares entre a produtividade de feijão e a porosidade do solo foram analisados: (a) a variabilidade do atributo da planta e do solo; (b) as correlações lineares e espaciais entre a produtividade de grãos de feijão e a macroporosidade, microporosidade e a porosidade total do solo; e (c) os atributos do solo na busca de um indicador da sua qualidade física, visando ao aumento da produtividade de grãos.

\section{MATERIAL E MÉTODOS}

O experimento foi desenvolvido em área pertencente à Faculdade de Engenharia de Ilha SolteiraUNESP, localizada no município de Selvíria (MS), com coordenadas geográficas de $51^{\circ} 22^{`}$ de longitude oeste e $20^{\circ} 22^{`}$ de latitude sul e altitude de $335 \mathrm{~m}$, no ano agrícola 2004/2005. O solo pesquisado foi um Latossolo Vermelho distroférrico (Embrapa, 2006). Foram analisados o atributo da planta (produtividade de grãos) e os do solo (macroporosidade, microporosidade, e porosidade total, em quatro profundidades: $1(0,0-0,10 \mathrm{~m}), 2(0,10-0,20 \mathrm{~m}), 3(0,20-0,30 \mathrm{~m}) \mathrm{e}$ $4(0,30-0,40 \mathrm{~m})$. Para tal, foi instalada uma rede geoestatística experimental constituída de 75 pontos ordenados a cada $10 \mathrm{~m}$, distribuídos numa área de $50 \times 150 \mathrm{~m}$. Também foram amostrados 60 pontos distribuídos em quatro refinamentos alocados aleatoriamente na grande malha, visando detalhar o estudo da dependência espacial dos dados para condições menores que $10 \mathrm{~m}$. O refinamento ficou constituído por seis transeções paralelas às transeções da grande malha, totalizando 15 pontos amostrais em cada refinamento. Cada nova transeção adicionou mais 15 pontos amostrais, totalizando 135 pontos. A análise descritiva inicial, a matriz de correlação (simples e múltipla) e a análise de regressão entre os atributos estudados foram efetuadas com o SAS (Schlotzhaver \& Little, 1997). Já a análise geoestatística foi realizada pelo uso do Gamma Design Software 7.0 (GS+, 2004). Aos atributos que apresentaram tendências nos seus dados foi aplicada a técnica do refinamento pela mediana, conforme preconizado por Silva et al. (2003), obtendo-se os respectivos resíduos. Também os atributos sucedidos das letras a e $b$ tiveram valores representados pelos logaritmos decimais, respectivamente divididos por 10 e 100, enquanto o símbolo \# significou que foram utilizados os resíduos do atributo.

\section{RESULTADOS E DISCUSSÃO}

No quadro 1 constam as análises descritivas iniciais da produtividade de grãos de feijão e da porosidade do solo. A produtividade de grãos (Quadro 1) apresentou valor médio de $2.752 \mathrm{~kg}$ ha $^{-1}$, ficando tal valor acima da produtividade média do Estado, conforme Embrapa (2007), e superior àquela relatada por FAO (2007),

Quadro 1. Análise descritiva inicial da produtividade de grãos de feijão e da porosidade num Latossolo Vermelho distroférrico de Selvíria (MS)

\begin{tabular}{|c|c|c|c|c|c|c|c|c|c|c|}
\hline \multirow{3}{*}{ Atributo } & \multicolumn{10}{|c|}{ Medidas estatísticas descritivas } \\
\hline & \multirow[t]{2}{*}{ Média } & \multirow{2}{*}{ Mediana } & \multicolumn{2}{|c|}{ Valor } & \multirow{2}{*}{ Desvio-padrão } & \multicolumn{3}{|c|}{ Coeficiente } & \multicolumn{2}{|c|}{$\begin{array}{l}\text { Probabilidade } \\
\text { do teste }\end{array}$} \\
\hline & & & Mínimo & Máximo & & Variação & Curtose & Assimetria & $\operatorname{Pr}<\mathbf{w}$ & DF \\
\hline \multirow{3}{*}{ PG $\left(\mathrm{kg} \mathrm{ha}^{-1}\right)$} & \multicolumn{10}{|c|}{$\begin{array}{c}\% \\
\text { Atributo da planta }\end{array}$} \\
\hline & 2,752 & 2,753 & 1,391 & 4,073 & 0,515 & 18,7 & $-0,112$ & $-0,030$ & 0,887 & $\mathrm{NO}^{(2)}$ \\
\hline & \multicolumn{10}{|c|}{ Atributo do solo } \\
\hline $\operatorname{MA1b}\left(\mathrm{m}^{3} \mathrm{~m}^{-3}\right)$ & 0,114 & 0,111 & 0,049 & 0,220 & 0,131 & 12,4 & $-0,189$ & $-0,113$ & 0,721 & $\mathrm{LN}^{(3)}$ \\
\hline $\operatorname{MA} 2 b\left(\mathrm{~m}^{3} \mathrm{~m}^{-3}\right)$ & 0,098 & 0,098 & 0,051 & 0,205 & 0,116 & 11,7 & 0,045 & 0,072 & 0,524 & $\mathrm{LN}$ \\
\hline MA3 $\left(\mathrm{m}^{3} \mathrm{~m}^{-3}\right)$ & 0,100 & 0,099 & 0,023 & 0,179 & 0,028 & 27,6 & 0,321 & 0,314 & 0,389 & NO \\
\hline MA4 $\left(\mathrm{m}^{3} \mathrm{~m}^{-3}\right)$ & 0,117 & 0,115 & 0,046 & 0,224 & 0,034 & 29,2 & $-0,123$ & 0,203 & 0,468 & NO \\
\hline $\operatorname{MI} 1\left(\mathrm{~m}^{3} \mathrm{~m}^{-3}\right)$ & 0,339 & 0,340 & 0,293 & 0,390 & 0,021 & 6,3 & $-0,412$ & $-0,106$ & 0,386 & NO \\
\hline $\operatorname{MI} 2\left(\mathrm{~m}^{3} \mathrm{~m}^{-3}\right)$ & 0,346 & 0,344 & 0,304 & 0,407 & 0,019 & 5,5 & 0,134 & 0,245 & 0,543 & NO \\
\hline $\operatorname{MI} 3\left(\mathrm{~m}^{3} \mathrm{~m}^{-3}\right)$ & 0,359 & 0,357 & 0,282 & 0,406 & 0,024 & 6,6 & $-0,143$ & $-0,116$ & 0,215 & NO \\
\hline $\operatorname{MI} 4\left(\mathrm{~m}^{3} \mathrm{~m}^{-3}\right)$ & 0,364 & 0,368 & 0,301 & 0,408 & 0,023 & 6,4 & $-0,380$ & $-0,303$ & 0,216 & NO \\
\hline PT1a $\left(\mathrm{m}^{3} \mathrm{~m}^{-3}\right)$ & 0,458 & 0,454 & 0,401 & 0,559 & 0,026 & 3,9 & 0,371 & 0,554 & 0,034 & $\mathrm{TL}^{(4)}$ \\
\hline PT2 $\left(\mathrm{m}^{3} \mathrm{~m}^{-3}\right)$ & 0,447 & 0,449 & 0,372 & 0,502 & 0,024 & 5,4 & 0,111 & $-0,259$ & 0,214 & NO \\
\hline PT3 $\left(\mathrm{m}^{3} \mathrm{~m}^{-3}\right)$ & 0,459 & 0,461 & 0,380 & 0,536 & 0,024 & 5,3 & 0,641 & $-0,167$ & 0,640 & NO \\
\hline $\mathrm{PT} 4\left(\mathrm{~m}^{3} \mathrm{~m}^{-3}\right)$ & 0,482 & 0,484 & 0,405 & 0,554 & 0,027 & 5,6 & $-0,078$ & $-0,252$ & 0,402 & NO \\
\hline
\end{tabular}

(1) Pr: probabilidade do teste. ${ }^{(2)}$ NO: distribuição normal. ${ }^{(3)}$ LN: lognormal. (4) TL tendendo à lognormalidade. 
muito provavelmente devido à alta tecnologia empregada como tratos culturais e irrigação. Já com relação à microporosidade do solo, seus valores médios foram de $0,339 \mathrm{~m}^{3} \mathrm{~m}^{-3}$ (MI), $0,346 \mathrm{~m}^{3} \mathrm{~m}^{-3}$ (MI2), $0,359 \mathrm{~m}^{3} \mathrm{~m}^{-3}$ (MI3) e $0,364 \mathrm{~m}^{3} \mathrm{~m}^{-3}$ (MI4), seguindo um padrão linear positivo em relação ao aumento da profundidade. Tal fato ficou em consonância com os trabalhos de Suzuki \& Alves. (2006) e Carvalho et al. (2003), cujos dados também apresentaram o mesmo padrão, provavelmente devido ao tráfego de máquinas. Apesar de se tratar de uma área de plantio direto, esta foi anteriormente ocupada por culturas anuais por mais de 25 anos em sistema convencional com uso de arado e grade. Os valores de microporosidade encontrados ficaram muito próximos dos níveis preconizados por Baver (1973) e Azevedo \& Dalmolin (2006), isto é, $0,333 \mathrm{~m}^{3} \mathrm{~m}^{-3}$; valor acima deste pode afetar o crescimento das plantas.

Os valores de $0,458 \mathrm{~m}^{3} \mathrm{~m}^{-3}$ (PT1a), $0,447 \mathrm{~m}^{3} \mathrm{~m}^{-3}$ (PT2), 0,459 $\mathrm{m}^{3} \mathrm{~m}^{-3}$ (PT3) e $0,482 \mathrm{~m}^{3} \mathrm{~m}^{-3}$ (PT4) de porosidade do solo ficaram pouco abaixo do limite inferior estabelecido para o solo agrícola ideal preconizado por Kiehl (1979) e Azevedo \& Dalmolin (2006), que é de $0,500 \mathrm{~m}^{3} \mathrm{~m}^{-3}$. Esse fato pode ter perfeitamente contribuído para que houvesse uma diminuição na produção de grãos de feijão, não liberando, portanto, ainda que tenham sido utilizados a irrigação e todos os tratos necessários à cultura, a manifestação da sua máxima capacidade produtiva. Este fato provavelmente aconteceu novamente devido à intensa mecanização da área nos anos anteriores (Quadro 1).

Das correlações lineares e exponenciais entre o atributo PG e os do solo (Quadro 2), aquelas significativas foram os pares PG e MI1 $\left(\mathrm{r}=-0,207^{*}\right)$, PG e PT3 $\left(r=-0,209^{*}\right)$ e PG e MA1b $\left(r=0,224^{*}\right)$. O primeiro par apresentou correlação negativa entre causa e efeito. Já a PG e PT3, por apresentar correlação negativa entre os atributos, torna tal fato de difícil explicação e ainda discorda de Santos et al. (2006), que não encontraram significância para esse par. No entanto, se for admitido o fato de que provavelmente esteja faltando contato entre a raiz e o solo, quando comparado o mapa de co-krigagem (Figura 3b), foram observadas, em relação aos mapas de krigagem simples, as maiores produtividades de grãos de feijão (Figura 1b) nos mesmos sítios onde ocorreram as menores porosidades (Figura $2 \mathrm{~b}$ ) para a profundidade de 0,10-0,20 m. Dessa forma, a suposição inicial da falta de contato raiz/solo pode-se tornar um argumento plausível para tal explicação. Já o último par (PG e MA1b) denotou função crescente entre causa e efeito, o que ficou de acordo com Carvalho et al. (2002), que observaram o mesmo padrão para a macroporosidade do solo, quando correlacionada com a produtividade de grãos de feijão. Assim, a PG pôde ser estimada pelas seguintes equações, em função MI1 (regressão linear), MA1b e PT3 (regressão exponencial), respectivamente:

$\mathrm{PG}=4,455-5,025^{*} \mathrm{MI} 1\left(\mathrm{R}^{2}=0,207, \mathrm{p}<0,05\right)$

$\mathrm{PG}=1,894 \mathrm{e}^{0,1268^{* *} \mathrm{MA1b}}\left(\mathrm{R}^{2}=0,224, \mathrm{p}<0,01\right)$

$\mathrm{PG}=5,855 \mathrm{e}^{1,684^{*} \mathrm{PT} 3} \quad\left(\mathrm{R}^{2}=0,209, \mathrm{p}<0,05\right)$

Em relação ao atributo da planta $(\mathrm{PG})$, quando estabelecido como variável dependente, aos do solo (MA2b, MI2 e PT2) como variáveis independentes (Quadro 2), foi ajustada a seguinte equação de regressão linear múltipla:

$$
\begin{gathered}
\mathrm{PG}=1,229+6,504^{* *} \mathrm{MA} 2 \mathrm{~b}+ \\
2,974.10^{* * *} \mathrm{MI} 2-27,44^{* *} \mathrm{PT} 2 \\
\left(\mathrm{R}^{2}=0,214, \mathrm{p}<0,01\right)
\end{gathered}
$$

em que PG é a produtividade de grãos de feijão $\left(t^{h} a^{-1}\right)$, MA1b, MI1 e PT3 representam a macroporosidade e a microporosidade, ambas na primeira camada, e a porosidade total na terceira camada do solo $\left(\mathrm{m}^{3} \mathrm{~m}^{-3}\right)$, e MA2b, MI2 e PT2 representam a macroporosidade, a microporosidade e a porosidade total, respectivamente, na segunda camada do solo $\left(\mathrm{m}^{3} \mathrm{~m}^{-3}\right)$.

\begin{tabular}{|c|c|c|c|c|c|c|c|c|c|c|c|c|}
\hline & PG & MA1b & MA2b & MA3 & MA4 & MI1 & MI2 & MI3 & MI4 & PT1a & PT2 & PT3 \\
\hline MA1b & 0,224 * & & & & & & & & & & & \\
\hline MA2b & $0,088^{\mathrm{ns}}$ & $0,283 * *$ & & & & & & & & & & \\
\hline MA3 & $-0,068^{\mathrm{ns}}$ & $0,115^{\mathrm{ns}}$ & $0,273 * *$ & & & & & & & & & \\
\hline MA4 & $0,014^{\mathrm{ns}}$ & $0,251^{* *}$ & $0,250 * *$ & $0,255^{* *}$ & & & & & & & & \\
\hline MI1 & $-0,207^{*}$ & $-0,560 * *$ & $-0,165^{\mathrm{ns}}$ & $-0,156^{\mathrm{ns}}$ & $-0,125^{*}$ & & & & & & & \\
\hline MI2 & $-0,104^{\mathrm{ns}}$ & $-0,216^{*}$ & $-0,447 * *$ & $-0,298 * *$ & $-0,124^{\mathrm{ns}}$ & $0,592^{* *}$ & & & & & & \\
\hline MI3 & $-0,049^{\mathrm{ns}}$ & $-0,025^{\mathrm{ns}}$ & $-0,085^{\mathrm{ns}}$ & $-0,475 * *$ & $-0,022^{\mathrm{ns}}$ & $0,559 * *$ & $0,671^{* *}$ & & & & & \\
\hline MI4 & $-0,094^{\mathrm{ns}}$ & $-0,150^{\mathrm{ns}}$ & $-0,118^{\mathrm{ns}}$ & $-0,245^{* *}$ & $-0,521 * *$ & $0,518 * *$ & $0,585 * *$ & $0,619 * *$ & & & & \\
\hline PT1a & $0,064^{\mathrm{ns}}$ & $0,749 * *$ & $0,186^{*}$ & $-0,045^{\mathrm{ns}}$ & $0,099^{\mathrm{ns}}$ & $-0,038^{\mathrm{ns}}$ & $0,235^{* *}$ & $0,287 * *$ & $0,195^{*}$ & & & \\
\hline PT2 & $-0,088^{\mathrm{ns}}$ & $0,072^{\mathrm{ns}}$ & $0,696 * *$ & $0,054^{\mathrm{ns}}$ & $0,130^{\mathrm{ns}}$ & $0,279 * *$ & $0,282 * *$ & $0,385 * *$ & $0,310 * *$ & $0,370 * *$ & & \\
\hline PT3 & $0,209 *$ & $-0,012^{\mathrm{ns}}$ & $0,152^{\mathrm{ns}}$ & $0,608 * *$ & $0,201^{*}$ & $0,290 * *$ & $0,368 * *$ & $0,306 * *$ & $0,320 * *$ & $0,235 * *$ & $0,464 * *$ & \\
\hline $\mathrm{PT} 4$ & $-0,129^{\mathrm{ns}}$ & $0,087^{\mathrm{ns}}$ & $0,138^{\mathrm{ns}}$ & $0,055^{\mathrm{ns}}$ & $0,718 * *$ & $0,167^{\mathrm{ns}}$ & $0,384 * *$ & $0,469 * *$ & $0,114^{\mathrm{ns}}$ & $0,278 * *$ & $0,442 * *$ & $0,533 * *$ \\
\hline
\end{tabular}

Quadro 2. Matriz de correlação linear simples entre a produtividade de grãos de feijão e a porosidade num Latossolo Vermelho distroférrico de Selvíria (MS)

*: Indica interação, relação entre variáveis independentes. 
A análise geoestatística das krigagens (Quadro 3) evidenciou semivariogramas, com uma série de pontos (lags) bem ajustados à função, tanto para a planta quanto para o solo. O melhor foi ajustado para o\#PT2 (Figura 2a), com um coeficiente de determinação espacial de 0,945, enquanto para a PG (Figura 1a) foi de 0,825 . Todos os atributos pesquisados apresentaram dependência espacial, exceto o MA2b e o \#MI4. Os demais atributos evidenciaram que suas distribuições no espaço não foram aleatórias, uma vez que a classe de dependência espacial da PG foi de 76,9 \% (alta), e do solo variou entre 50,1 \% (moderada/MA3) e 90,4 \% (alta/\#MI2), concordando tal fato com os trabalhos de Carvalho et al. (2002, 2003), Souza et al. (2004) e Santos et al. (2006), que apresentaram classes semelhantes de dependência espacial para os mesmos atributos do solo. Portanto, neste estudo, pôde-se constatar que $76,9 \%$ da variação total da $P G$ foi explicada pela dependência espacial, enquanto o efeito pepita atribuído ao erro casual foi de 23,1\%.

$\mathrm{Na}$ análise das co-krigagens (Quadro 3), o enfoque foi para a $\mathrm{PG}=\mathrm{f}(\# \mathrm{PT} 2)$, uma vez que este apresentou semivariograma cruzado com lags distribuídos apenas em um dos quadrantes (quarto) (Figura 3a), denotando, assim, um aspecto confiável entre as variáveis \#PT2 e PG, apresentando, ainda, um confiável $\mathrm{R}^{2}=0,676$. Assim, em sua krigagem simples (Figuras $1 \mathrm{~b}$ e $2 \mathrm{~b}$ ), em que ocorreu um aumento da porosidade total do solo na segunda camada (Regiões Centro-oeste, Centro-sul e Sudeste), com valores entre 0,060 e $0,076 \mathrm{~m}^{3} \mathrm{~m}^{-3}$, a $\mathrm{PG}$ variou entre $2.279 \mathrm{e}$ $2.064 \mathrm{~kg} \mathrm{ha}^{-1}$, com média de $2.172 \mathrm{~kg} \mathrm{ha}^{-1}$. Desse (a)

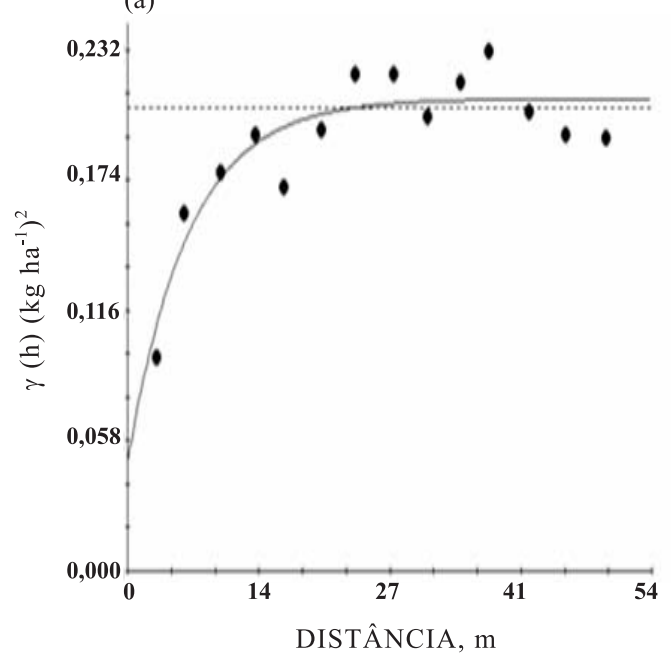

(b)

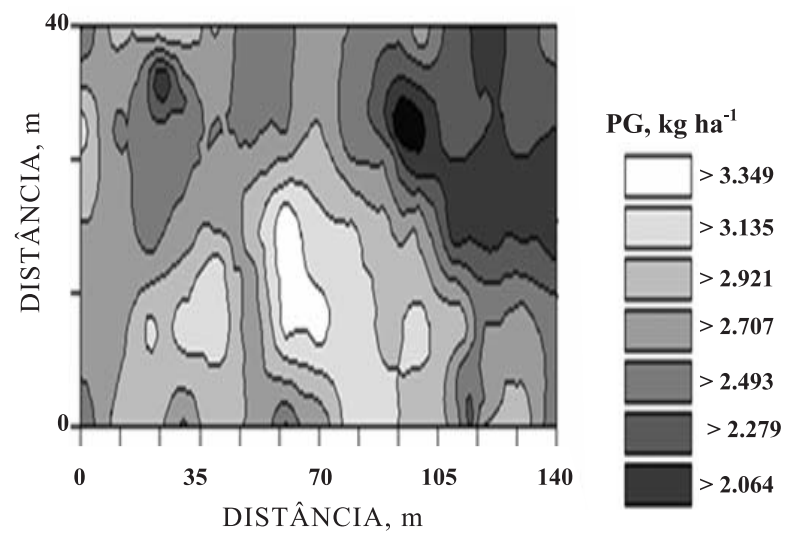

Figura 1. Semivariograma simples da produtividade de grãos de feijão (PG) (a); e mapa de krigagem da produtividade (PG) de um Latossolo Vermelho distroférrico de Selvíria (MS) (b).

(a)

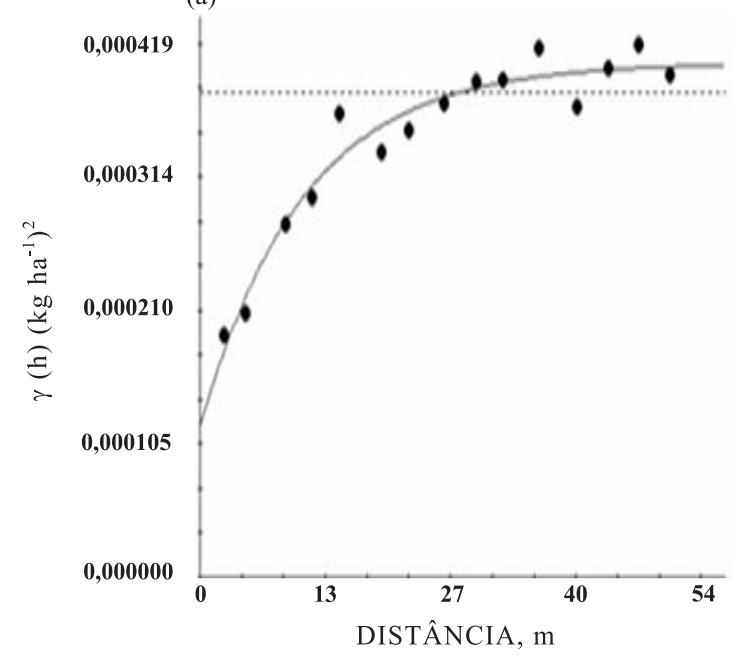

(b)

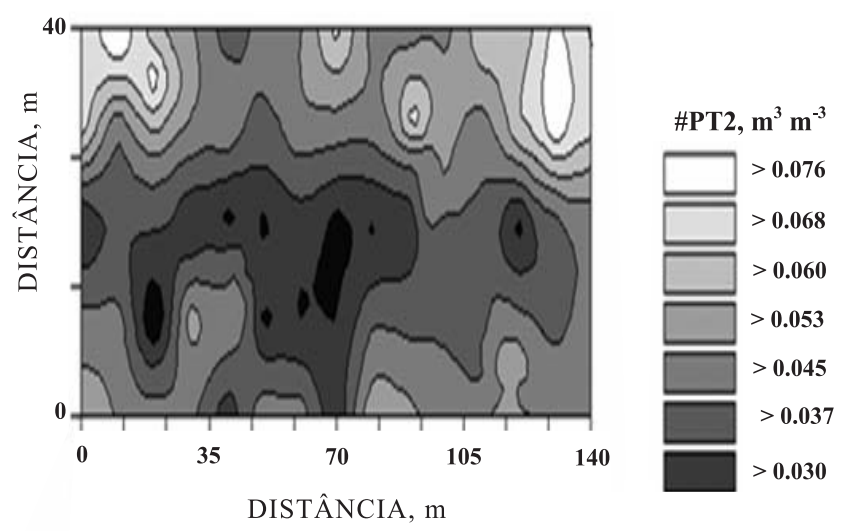

Figura 2. Semivariograma simples da porosidade total do solo (\#PT2) (a); e mapa de krigagem da porosidade total (\#PT2) de um Latossolo Vermelho distroférrico de Selvíria (MS) (b). 


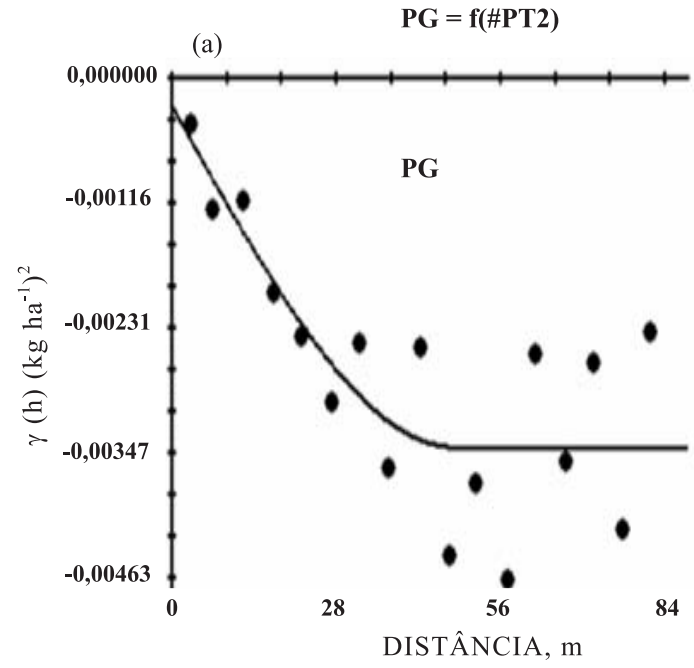

(b)

Figura 3. Semivariograma cruzado entre a produtividade de grãos de feijão (PG) e a porosidade total (\#PT2) de um Latossolo Vermelho distroférrico de Selvíria (MS) (a); e mapa de co-krigagem referente à PG = f(\#PT2) (b).

Quadro 3. Parâmetros dos semivariogramas ajustados para a produtividade de grãos de feijão, macroporosidade, microporosidade e porosidade total de um Latossolo Vermelho distroférrico de Selvíria (MS)

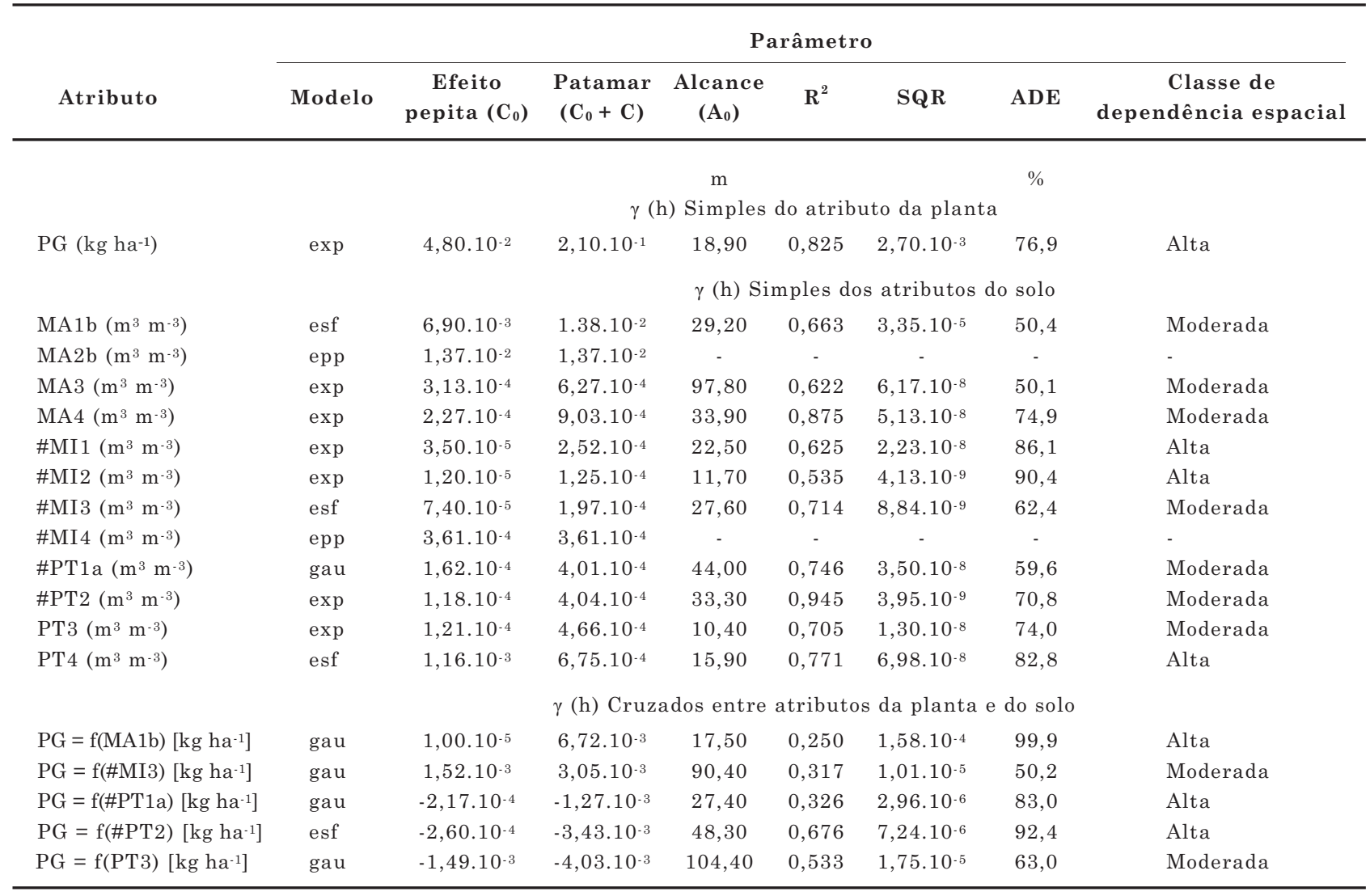

modo, o padrão desses dados discordou dos de Santos et al. (2006), que apresentaram mapas de krigagem, indicando que, nas áreas de menor porosidade do solo, a produtividade da planta foi menor, e vice-versa, nas mesmas condições deste experimento. Em contrapartida, onde ocorreu uma substancial 
diminuição da porosidade total do solo na segunda camada (Regiões Norte, Nordeste e Centro-Sul), com valores entre 0,037 e $0,030 \mathrm{~m}^{3} \mathrm{~m}^{-3}$, a $\mathrm{PG}$ variou entre 2.921 e $3.349 \mathrm{~kg} \mathrm{ha}^{-1}$, com média de $3.135 \mathrm{~kg} \mathrm{ha}^{-1}$. Com relação ao referido par, notou-se que seus mapas de krigagem possuíram uma harmônica e inversa proporcionalidade, sendo tal fato de difícil explicação. No entanto, estudos mostraram que solos excessivamente porosos são prejudiciais à absorção de água e nutrientes pelas raízes, por causa do menor contato raiz-solo, proporcionando também menor desenvolvimento das plantas. Portanto, essa aproximação dos agregados, que ocorreu na porosidade total de 0,10-0,20 m, melhorou este contato, propiciando a passagem de poros de elevado diâmetro para aqueles que correspondam a uma tensão de retenção de água dentro da capacidade de água disponível, concordando com os trabalhos de Dias Junior \& Pierce(1996); Kooistra et al. (1992); Hakansson et al. (1998), que também verificaram a necessidade dessa maior aproximação entre solo e raiz.

\section{CONCLUSÕES}

1. Os atributos estudados não variaram aleatoriamente: apresentaram variabilidade entre média e baixa dos dados e seguiram padrões espaciais bem definidos, com alcance entre 11,70 e 104,40 m.

2. A correlação linear entre a produtividade de grãos de feijão irrigado com os atributos do solo foi baixa, embora altamente significativa. As de melhor correlação com a PG foram a MA1b e MI1.

3. Do ponto de vista espacial, houve excelente correlação inversa entre a PG e\#PT2. Portanto, nos sítios onde a \#PT2 aumentou $\left(0,060-0,076 \mathrm{~m}^{3} \mathrm{~m}^{-3}\right)$, a $\mathrm{PG}$ variou de 2.279 a $2.064 \mathrm{~kg} \mathrm{ha}^{-1}$, já naqueles onde diminuiu $\left(0,037-0,030 \mathrm{~m}^{3} \mathrm{~m}^{-3}\right)$, a PG ficou de $2.921 \mathrm{e}$ $3.349 \mathrm{~kg} \mathrm{ha}{ }^{-1}$.

\section{LITERATURA CITADA}

AZEVEDO, A.C. \& DALMOLIN, R.S.D. Solos e ambiente: Uma introdução. 2.ed. Santa Maria, Pallotti, 2006. 100p.

BAVER, L.D.; GARDNER, W.J. \& GARDNER, W.R. Física de suelos. México, Unión Tipográfica Editorial HispanoAmericana, 1973.

CAMARGO, A.M.M.P.; CASER, D.V. \& FRANCISCO, V.L.F.S Previsões e estimativas das safras agrícolas no Estado de São Paulo. Inf. Econ., 31:101-117, 2001.

CARVALHO, M.P.; SORATTO, R.P. \& FREDDI, O.S. Variabilidade espacial de atributos físicos em um Latossolo Vermelho distrófico sob preparo convencional em Selvíria, Estado de Mato Grosso do Sul. Acta Sci., 24:1353-1361, 2002.
CARVALHO, M.P.; TAKEDA, E.Y. \& FREDDI, O.S. Variabilidade espacial de atributos de um solo sob videira em Vitória Brasil (SP). R. Bras. Ci. Solo, 27:695-703, 2003.

DIAS JÚNIOR, M.S. \& PIERCE, F.J. O processo de compactação do solo e sua modelagem. R. Bras. Ci. Solo, 20:175-182, 1996.

EMPRESA BRASILEIRA DE PESQUISA AGROPECUÁRIA EMBRAPA. Sistema brasileiro de classificação de solos. Rio de Janeiro, Embrapa/CNPSo, 2006. 306p.

EMPRESA BRASILEIRA DE PESQUISA AGROPECUÁRIA . EMBRAPA. Disponível em: <http://www.cnpaf.embrapa.br. Acesso em: 15 Abril de 2007.

FNP-CONSULTORIA \& COMERCIO. Anuário da Agricultura Brasileira - AGRIANUAL 2007. Feijão. São Paulo, 2007.

GOEDERT, W.J.; SCHERMACK, M.J. \& FREITAS DE, F.C. Estado de compactação do solo em áreas cultivadas no sistema de plantio direto. Pesq. Agropec. Bras., 37:223$227,2002$.

GS+: Geostatistics for environmental sciences. 7.ed. Plainwell, Gamma Desing Software, 2004. 159p.

HAKANSSON, I.; STENBERG, M. \& RYDBERG, T. Long term experiments with different depths of mouldboard plough in Sweden. Soil Till. Res., 46:209-223, 1998.

KERTZMANN, F.F. Modificações na estrutura e no comportamento de um Latossolo Roxo provocados pela compactação. São Paulo, Universidade de São Paulo, 1996. 153p. (Tese de Doutorado)

KIEHL, E.J. Manual de edafologia: Relações solo-planta. Piracicaba, Agronômica Ceres, 1979. 264p.

KOOISTRA, M.J.; SCHOONDERBEEK, D.; BOONE, F.R.; VEEN, B.W. \& van NOORDWIJK, M. Root soil contact of maize, as measured by a thin section technique - II: Effects of soil compaction. Plant Soil, 139:119-129, 1992.

ORGANIZAÇÃO DAS NAÇÕES UNIDAS PARA AGRICULTURA E ALIMENTAÇÃO. (FAO). Disponível em: <http://www.fao.org> Acesso em: 10 abril de 2007.

SANTOS, M.L.; CARVALHO, M.P.; RAPASSI, R.M.A.; MURAISHI, C.T.; MALLER, A. \& MATOS, F.A. Correlação linear e espacial entre produtividade de milho (Zea mays L.) e atributos físicos de um Latossolo Vermelho distroférrico sob plantio direto do Cerrado Brasileiro. Acta Sci., 28:313-321, 2006.

SCHLOTZHAVER, S.D. \& LITTLE, R.C. SAS - System for elementary statistical analysis. 2.ed. Cary, 1997.441p.

SILVA, V.R.; REICHERT, J.M.; STORCK, L. \& FEIJÓ, S. Variabilidade espacial das características químicas do solo e produtividade de milho em um Latossolo VermelhoAmarelo distrófico arênico. R. Bras. Ci. Solo, 27:10131020, 2003. 
SOUZA, Z.M.; MARQUES JÚNIOR, J.; PEREIRA, G.T. \& BENTO, M.J.C. Variabilidade espacial de atributos físicos de um Latossolo Vermelho sob cultivo de cana-de-açúcar. R. Bras. Eng. Agríc. Amb., 8:51-58, 2004.
SUZUKI, L.E.A.S. \& ALVES, M.C. Fitomassa de plantas de cobertura em diferentes sucessões de culturas e sistemas de cultivo. Bragantia, 65:121-127, 2006. 